genetic dysregulation associated with these various syndromes, and environmental risk factors, cause abnormalities in neuronal migration and synaptic development, leading to defects in neurotransmitter metabolism and cortical organization. The resulting deficits in attention, motor control and executive function are characteristic of ADHD. ADHD is the final behavioral and cognitive phenotype in a cascade resulting from the genetic syndrome and factors in the environment. (Lo-Castro A, D'Agati E, Curatolo P. ADHD and genetic syndromes. Brain Dev June 2011;33:456-461). (Response: Adriana LoCastro, Department of Neuroscience, Pediatric Neurology Unit, "Tor Vergata" University of Rome-Italy, Viale Oxford, 81, 00133, Rome, Italy. E-mail: a.locastro(alibero.it).

COMMENT. New genetic techniques have identified several genes and syndromes associated with the behavioral and cognitive phenotype of ADHD. A genomewide analysis of large, rare chromosomal deletions and duplications known as copy number variants (CNVs) in 366 children with ADHD and matched controls, aged 5-17 years, showed a significantly increased rate of CNVs in ADHD children, especially in those with intellectual disability. An excess of chromosome 16p13.11 duplications was noted in the ADHD group. (Williams NM, et al. Lancet Oct 23, 2010;376:1401-1408). It is interesting that the chromosome $16 \mathrm{p} 13$ region is involved in tuberous sclerosis, and epilepsy or epileptiform EEG is commonly associated with both TSC and ADHD. The identification of genetic syndromes associated with symptoms of ADHD may lead to more specific therapies.

\title{
EXECUTIVE AND INTELLECTUAL FUNCTIONS IN ADHD
}

The relationship between executive functions (EF), ADHD subtypes, and comorbid diagnoses, and the potential role of IQ were investigated in the Child Neuropsychiatry Unit, Children's Hospital, Rome, Italy. In a cohort of 23 children (aged 5 to 16 years, 20 males and 3 females) with a diagnosis of ADHD, no differences were found between children with different subtypes of ADHD on measures of EF. Differences on EF measures were observed in children with different comorbidities (internalizing vs externalizing disorders). Compared to the internalizing disorders group (depression, phobia, and anxiety), children with externalizing disorders (oppositional defiant or conduct disorders) obtained significantly lower scores on different measures of EF (i.e. verbal working memory and categorical fluency), but the differences were dependent on IQ level. Children with externalizing disorders exhibited significantly lower IQ scores than those with internalizing disorders. Comorbidity patterns, rather than ADHD subtypes, define the neuropsychological features of the ADHD phenotype. General IQ plays a role in influencing the cognitive processes underlying the disorder. (Di Trani M, Casini MP, Capuzzo F, et al. Executive and intellectual functions in attention-deficit/hyperactivity disorder with and without comorbidity. Brain Dev June 2011;33:462-469). (Response: Maria Pia Casini, Child Neuropsychiatry Unit, Children's Hospital Bambino Gesu, Piazza Sant'Onofrio 4, I-00165 Rome, Italy. E-mail: mariap.casini@opgb.net).

COMMENT. In an Editorial, Inagaki M (Brain Dev 2011;33:454-455) discusses the problems in executive function (EF) experienced by children with ADHD. The 
cognitive functions involved in goal-directed behavior are impaired, including inhibition, planning, set-shifting, working memory, and fluency, and reflect a dysfunction of frontosubcortical pathways. The neuropsychological deficits in ADHD are heterogeneous. Although the majority of ADHD children have a deficit on at least one EF measure, the same is true for nearly half a control group (Nigg JT et al, 2005). Different populations of children with neuro-developmental disorders have deficits in EF similar to those found in children with ADHD (Sergeant JA et al, 2002). The EF deficits with ADHD are nonspecific and not diagnostic. In the Multimodal Treatment Study of Children with ADHD, a 14-month trial of treatment strategies (1999), 40\% of the sample $(n=579)$ had a comorbid ODD that could modulate or interact with the intellectual and executive profile. The role of IQ in prognosis of ADHD alluded to in this review is well known. Risk factors for a poor prognosis include a low average or borderline IQ, and comorbid oppositional and conduct disorders.

\section{ATTENTION DEFICITS FOLLOWING ADEM RELIEVED BY GUANFACINE}

Neuroscientists at the Institute of Cognitive Neuroscience, London, UK report the case of a 38-year-old male with severe deficits in arousal and sustained attention, associated with hemispatial neglect, secondary to acute disseminated encephalomyelitis. Treatment with the noradrenergic agonist guanfacine was associated with significant improvements in both sustained attention and spatial neglect. Presenting with a right hemiparesis following a prodrome of headache and fever, he developed tonic-clonic seizures soon after admission. MRI revealed signal changes in the thalamus, cerebellum, and temporal and occipital lobes. CSF was normal, and EEG was consistent with encephalopathy and ADEM. Following courses of iv methylprednisole, immunoglobulin, plasma exchange and antibiotics, seizures at first refractory to AEDs were stabilized after 4 months. Neuropsychological testing revealed severe cognitive impairments and leftsided neglect. At a 2-year follow-up, he had persistent left hemiparesis with severe left hemispatial negect, and impairments in sustained attention and arousal. MRI showed lesions localized to the right thalamus and pulvinar, areas linked to the mechanism of neglect. This is the first demonstration of a persistent amelioration of spatial neglect following treatment with guanfacine. General level of alertness and arousal also improved. (Singh-Curry V, Malhotra P, Farmer SF, Husain M. Attention deficits following ADEM ameliorated by guanfacine. J Neurol Neurosurg Psychiatry June 2011;82:688-690). (Response: Professor Masud Husain, Institute of Cognitive Neuroscience, 17 Queen Square, London WC1N 3AR, UK. E-mail: m.husain@ion.ucl.ac.uk).

COMMENT. Impairments of arousal or maintained alertness are considered a component of the neglect syndrome, and arousal is dependent on noradrenergic input, responsive to guanfacine. Guanfacine, as a sustained release formulation, Intuniv, is effective in the treatment of ADHD (Biederman J et al. Pediatrics 2008;121:E73-84).

Hemispatial neglect is usually reported following stroke involving the medial thalamus and pulvinar in the right hemisphere. ADEM is a rare cause of neglect but is associated with impaired arousal and sustained attention, components of the neglect 